\title{
Assimilation and contrast illusions: Differences in plasticity
}

\author{
JOAN S. GIRGUS \\ Princeton University, Princeton, New Jersey \\ and \\ STANLEY COREN \\ University of British Columbia, Vancouver, British Columbia, Canada
}

\begin{abstract}
Three experiments show differences in the plasticity of the contrast and assimilation portions of the Delboeuf, Ebbinghaus, and Ponzo illusions. Contrast illusions show decrement in illusion magnitude with free inspection, whereas assimilation illusions do not. A model to explain both the original distortion and the differential susceptibility of the two classes of illueion to decrement is offered.
\end{abstract}

There are a number of visual stimulus configurations in which a particular component can be distorted in opposite directions, depending on the context in which it is embedded. When the test component is distorted in the direction of the surrounding context, the configuration is usually referred to as an assimilation illusion, because the perceived difference between the test and inducing elements is smaller than the actual difference and thus the apparent difference between test and context is reduced. When the direction of distortion is away from the surrounding context, the configuration is usually referred to as a contrast illusion, because the perceived difference between the test and inducing elements is larger than the actual difference and thus the apparent difference between them is accentuated (Coren \& Girgus, 1978).

The Delboeuf illusion, presented in Figure 1A, shows both types of distortion. When the concentric circle surrounding the central circle is just slightly larger than the central circle, as in the figure on the left, the central circle tends to be overestimated relative to the undistorted circle on the right, making this an assimilation illusion. When the concentric circle is much larger than the central circle, as in the middle figure, the central circle tends to be underestimated, thus forming a contrast illusion.

Figure 1B shows the Ebbinghaus illusion, which is frequently described as a pure contrast illusion because

This research was supported in part by grants from the National Sciences Research and Engineering Council of Canada and the National Science Foundation of the United States (74-18599). It represents the equal and shared contributions of both authors. J. S. Girgus's mailing address is: Department of Psychology, Princeton University, Princeton, New Jersey 08544. That of S. Coren is: Department of Psychology, University of British Columbia, Vancouver, British Columbia V6T 1W5, Canada. the central circle is seen as larger than it really is when surrounded by small circles and smaller than it really is when surrounded by larger circles. However, there is some indication in the literature that the distance between the central circle and either the centers or the outer edges of the surrounding circles may play a role in the judgment of the apparent size of the central circle (Cooper \& Weintraub, 1970; Girgus, Coren, \& Agdern, 1972). If this is the case, then the figure on the left should be viewed as a central circle surrounded by a nearby outer ring and the figure in the middle should be viewed as a central circle surrounded by a farther away outer ring. Obviously, this description bears a close resemblance to the Delboeuf illusion: the figure on the left, with its
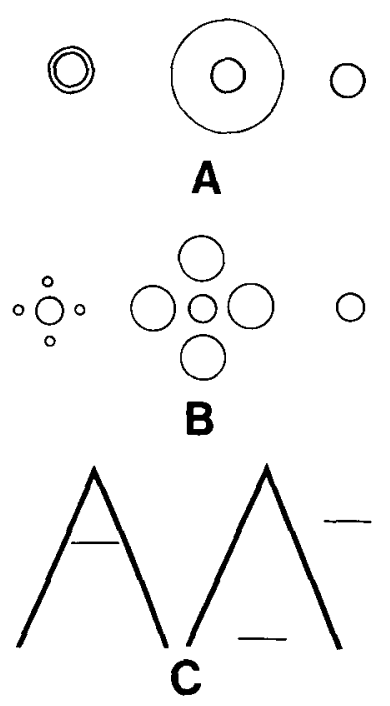

Figure 1. (A) The components of the Delboeuf illusion. (B) The components of the Ebbinghaus illusion. (C) The components of the Ponzo illusion. 
overestimated central circle, is the assimilation version, and the figure on the right, with its underestimated central circle, is the contrast version.

Figure 1C shows the Ponzo illusion, in which the apparent length of the horizontal line within the angle is distorted. The direction of the distortion seems to depend on the relative proximity of the ends of the line to the sides of the angle. Thus, the configuration on the left, in which the ends of the line are relatively close to the sides of the angle, is seen as longer than it really is, making this configuration an assimilation illusion. On the other hand, in the configuration on the right, in which the ends of the line are relatively farther from the sides of the angle, the line is seen as shorter than it really is, making this configuration a contrast illusion.

Although such size distortions have been known since the 1700s when Smith (1738) first noted that persons standing in front of large mountains or buildings tend to appear to be reduced in size, the mechanism responsible for such distortions has not yet been isolated. A variety of theories has been offered to explain both forms of distortion. Unfortunately, although both may occur in the same configuration, most theories deal only with either contrast or assimilation effects. This may well reflect a growing body of data that suggests that contrast and assimilation effects possibly represent separate classes of distortion (Coren, Girgus, Erlichman, \& Hakstian, 1976; Erlebacher \& Sekular, 1969; Quina \& Pollack, 1972).

Explanations of contrast illusions generally depend upon evocation of some form of cognitive-judgmental mechanism. Perhaps the first such explanation was offered by Helmholtz (1866/1962), who stated that, as a general principle, clearly perceived sensory differences tend to be exaggerated. This suggested to him that a variety of different types of cognitive contrast might exist, including size contrast, directional contrast, and even shape contrast. This theorem was elaborated by Wundt (1894), who called it the "law of perceptual relativity." Helson (1964) offered a general contrast principle, which was susceptible to mathematical treatment, in his "adaptation level theory." Since that time, several quantitative treatments of contrast have been offered (Massaro \& Anderson, 1971; Restle, 1971a; Restle \& Merryman, 1968).

It seems likely that the illusions found in size contrast configurations involve some contribution from cognitive-judgmental information processing strategies. Restle (1971b) reported that the magnitude of such size contrast illusions is reduced if the observer is instructed to ignore the surrounding context. Similarly, Coren and Miller (1974) found that the magnitude of the Ebbinghaus illusion varied as a function of the perceived similarity of the test and inducing items. Coren (1971) provides additional information that suggests a cognitive basis for size contrast illusions. He was able to demonstrate that contrast varies as a function of ap- parent rather than physical size of the inducers in the Ebbinghaus illusion.

Assimilation effects are usually explained by means of some form of averaging theory. For instance, Pressey $(1970,1971)$, who formalized and extended an idea proposed by Brunot (1893), suggested that observers form a global impression based upon the average of all extents within the part of the visual field to which they are attending and that therefore the apparent size of judged elements assimilates or regresses toward the average of these extents. Erlebacher and Sekular (1969) suggested that some size distortions are engendered by confusion between the test and inducing extents, which would lead to the same perceived distortion as an averaging theory.

As for contrast effects, there seems to be a cognitive basis for assimilation effects. Benussi (1904) and Coren and Girgus (1972a) have shown that some size illusions that have been explained as manifestations of assimilation can be attenuated when test and inducing elements are made discriminably different from each other, through the use of color or spatial separations. Furthermore, Coren and Girgus (1972a) were able to demonstrate that focusing an individual's attention on the test elements, to the exclusion of the inducing elements, results in reduction of such assimilation effects.

One method that has been used to investigate the contribution of cognitive strategies to visual geometric illusions is based upon the phenomenon known as illusion decrement. It has been frequently demonstrated that the magnitude of many illusions will diminish during a few minutes of free inspection. This effect has been shown in the Mueller-Lyer, Oppel-Kundt, Zoellner, Poggendorff, and Wundt-Hering illusions (Coren \& Girgus, 1972b; Coren \& Hoenig, 1972). It seems likely that this diminution or decrement in illusion magnitude involves a cognitive rather than a structural recalibration, since the effects are cumulative over days and weeks (Girgus, Coren, Durant, \& Porac, 1975; Judd, 1902). Furthermore, the rate of decrement varies as a function of traditional learning variables, such as the spacing of trials (Dewar, 1868; Mountjoy, 1958). Perhaps the strongest evidence for a cognitive basis for this diminution of illusion comes from Coren and Girgus (1974), who found that illusion decrement transfers from one configuration to another, with the magnitude of transfer varying as a function of the perceived rather than of the physical similarity between configurations, a result which has been replicated by Porac, Coren, Girgus, and Verde (1979).

To the extent that illusion decrement represents a change in the information processing or cognitive components of an illusion, it provides an interesting opportunity to assess whether similarities or differences between the assimilation and contrast components of illusions exist. Insofar as the assimilation and contrast distortions (which in this study involve overestimation and underestimation, respectively) in any given illusion 
are caused by similar information processing mechanisms, one would expect similar rates of recalibration and thus similar rates of illusion decrement. On the other hand, differences in the underlying cognitive mechanisms responsible for these two kinds of distortion could lead to differences in the rate of decrement with free inspection. In order to try to ascertain whether assimilation and contrast distortions have a common underlying basis, the following experiments involving illusion decrement were conducted.

\section{METHOD}

Three separate experiments that used different illusion configurations were conducted. Because of the similarity of methodology and procedure, the three experiments will be described together.

\section{Subjects}

Each of the three experiments employed 30 adult volunteers with $20 / 25$ or better Snellen acuity. Ten subjects were assigned to each of two experimental and one control condition in each experiment. Each subject was exposed to only one stimulus configuration.

\section{Stimuli and Apparatus}

Experiment 1. The first experiment involved the Delboeuf illusion. Figure $1 \mathrm{~A}$ shows the stimuli that were used. Both of the central test circles, as well as the control circle, were $14 \mathrm{~mm}$ in diameter ( $2 \mathrm{deg}$ of visual angle). The diameter of the concentric circle in the contrast, or apparently underestimated, portion of the configuration was $57 \mathrm{~mm}$ ( $8 \mathrm{deg} 6 \mathrm{~min}$ of visual angle). The diameter of the concentric circle in the assimilation, or apparently larger, portion was $19 \mathrm{~mm}$ ( $2 \mathrm{deg} 43 \mathrm{~min}$ ). All stimuli in this and the two succeeding experiments were drawn on white paper with black lines $1 \mathrm{~mm}$ wide; the reflectance of the lines was $2 \%$, and that of the background was $77 \%$. The light level averaged $95 \mathrm{~lx}$.

Judgments were made by rotating a wheel on which were drawn single comparison circles whose diameters ranged from 8.0 to $19.5 \mathrm{~mm}$ in $.5-\mathrm{mm}$ steps. The comparison circles appeared one at a time in a $26-\mathrm{mm}$ aperture cut into the apparatus.
Experiment 2. The second experiment involved the Ebbinghaus configuration. The stimuli used are shown in Figure 1B. The central test circles and the control circle were $14 \mathrm{~mm}$ in diameter. The diameter of each of the inducing circles in the contrast (underestimated) portion of the configuration was $23 \mathrm{~mm}$; in the assimilation (overestimated) half, the diameter of each of the surrounding circles was $5 \mathrm{~mm}$. The distance between the inner test circle and the inner edge of the four inducing circles was $6 \mathrm{~mm}$ in both experimental configurations. Thus, the total diameters of the under- and overestimated portions were $9 \mathrm{deg} 55 \mathrm{~min}$ and $4 \mathrm{deg} 51 \mathrm{~min}$ of visual angle, respectively. Judgments were made on the same apparatus used in Experiment 1.

Experiment 3. The third experiment involved the Ponzo illusion. The stimuli used are shown in Figure 1C. The size of the angle used was $45 \mathrm{deg}$, with $100-\mathrm{mm}(14 \mathrm{deg} 2 \mathrm{~min}$ of visual angle) sides. The horizontal line in the two illusion configurations and in the control figure was $30 \mathrm{~mm}$ (4 deg $17 \mathrm{~min}$ of visual angle). The test line was placed $55 \mathrm{~mm}$ up from the open end of the angle in the assimilation, or apparently larger, portion of the configuration. It was placed $3 \mathrm{~mm}$ up from the open end of the angle in the contrast, or apparently underestimated, portion of the illusion. Judgments were made using an adjustable line length set in a tongue-and-groove apparatus. .

\section{Procedure}

All three experiments used a common procedure. The stimuli were presented at a distance of $90 \mathrm{~cm}$ from the subject. For the Delboeuf and Ebbinghaus illusions, the subjects made an initial judgment of the size of the central (or control) circle, depending upon their assigned condition. Similarly, in the three Ponzo conditions, the subjects made an initial judgment of the length of the horizontal line. In all conditions, the subjects were instructed to scan the figure for a 5 -min period. Judgments of the apparent size of the test element were taken at 1 -min intervals during the inspection period.

\section{RESULTS}

The results of all three experiments are shown in Figure 2. The results from Experiment 1, involving the Delboeuf configuration, appear in Figure 2A. As expected, the initial judgment of the contrast portion of the configuration is significantly smaller than the initial

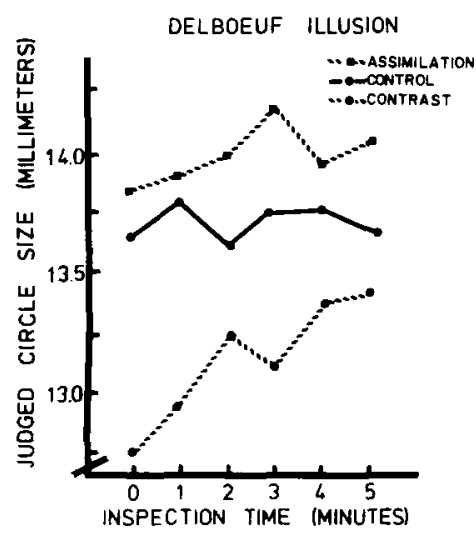

A

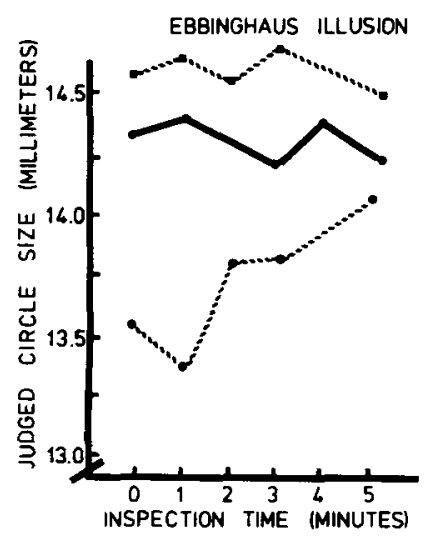

B

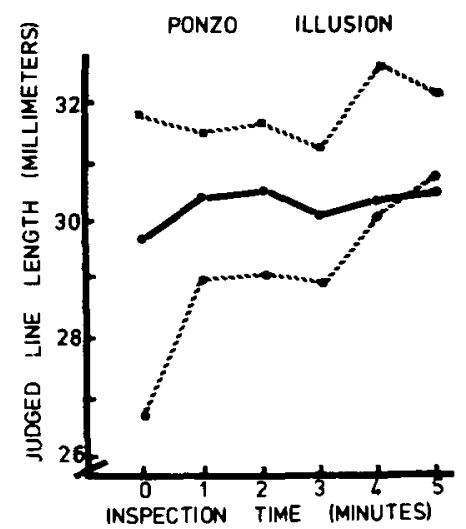

C

Figure 2. The magnitude of illusions as a function of time for the three experiments. (A) Experiment 1: the Delboeuf. (B) Experiment 2: the Ebbinghaus. (C) Experiment 3: the Ponzo. 
judgment of the control circle $[t(18)=5.61, \mathrm{p}<.01]$, and the initial judgment of the assimilation portion is significantly larger than the control circle $[t(18)=3.24$, $\mathrm{p}<.01]$. As expected, there is no significant change in the judgments of the size of the control configuration during the $5 \mathrm{~min}$ of inspection $[\mathrm{F}(5,45)=1.71]$. When we look at the contrast, or underestimated, portion of the configuration, we find that the illusion systematically diminishes or decrements (approaches the control level) during the viewing period $[\mathrm{F}(5,45)=7.94, \mathrm{p}<.01]$. For the assimilation, or overestimated, portion of the Delboeuf illusions, however, we find no significant change in magnitude $[F(5,45)=1.82]$, and hence no illusion decrement, during the inspection period. This difference between the assimilation and contrast decrement trends can also be seen in a statistically significant interaction $[F(5,90)=11.12, p<.01]$.

Experiment 2, involving the Ebbinghaus illusion, shows a remarkably similar pattern of results, as can be seen in Figure 2B. The initial measurements show a significant illusion, with the contrast portion of the configuration significantly underestimated $[\mathrm{t}(18)=5.39$, $\mathrm{p}<.01]$ and the assimilation portion significantly overestimated $[\mathrm{t}(18)=2.78, \mathrm{p}<.01]$. As expected, there is no significant change in the control configuration during the period of inspection $[F(5,45)=.38]$. For the illusory components, much the same pattern is found as was found in Experiment 1 for the Delboeuf illusion. The contrast segment of the illusion shows a significant reduction of the illusory effect during the inspection period $[F(5,45)=19.04, p<.01]$, but the assimilation portion of the configuration shows no significant change in illusion magnitude as a function of inspection $[F(5,45)$ $=1.03$ ]. Again, the interaction is statistically significant $[F(5,90)=12.44, p<.01]$.

The results of the third experiment, involving the Ponzo illusion, appear in Figure 2C. Again, these results are very similar to the results of the two previous experiments. As before, the initial measurements show significant illusion, with the contrast segment judgments significantly underestimated relative to the control group judgments $[t(18)=3.02, p<.01]$ and the assimilation segment significantly overestimated relative to the control configuration $[\mathrm{t}(18)=2.91, \mathrm{p}<.01]$. The control line shows no change as a function of inspection $[F(5,45)=1.33]$. As in the two previous experiments, the contrast portion of the illusion shows a significant diminution in illusion during the inspection period $[F(5,45)=4.66, p<.01]$. The assimilation portion of the illusion, however, shows no change as a function of viewing $[F(5,45)=1.89]$. As before, the interaction between the contrast and assimilation portions is significant $[F(5,90)=14.72, p<.01]$.

Despite the fact that each of these three experiments involved a different configuration, the results are strikingly similar. In each instance, we find a decrement or diminution of illusion magnitude for the contrast portion of the configuration but no significant changes in the assimilation portion.

\section{DISCUSSION}

The similarity in the patterns of illusion magnitude for the three configurations tested above strongly suggests that these configurations share common underlying mechanisms. The difference between the trends in the assimilation and contrast portions, the former showing no change and the latter showing the usual decrement, suggests a different mechanism for each of these two types of illusory distortion. This argues that any model proposed to. account for assimilation/contrast illusions must provide separate processes to account for these two kinds of effects. Such a model must contain an additional feature in that the mechanism suggested to account for the contrast illusions must be subject to recalibration during brief periods of free inspection, whereas the mechanism suggested to account for the assimilation effect must not be subject to such recalibration.

We propose that the data described above can be explained best by the pool-and-store model (Coren \& Girgus, 1978), which is based upon some normal judgmental processes involved in perception and which seems capable of accounting both for the phenomena of assimilation and contrast illusions and for the plasticity in the latter and lack of plasticity in the former. The basic data that lead to the pool-and-store model are those that demonstrate sequential dependencies in psychophysical judgments. Ward and Lockhead $(1970,1971)$ have shown, both for judgments of loudness and for judgments of line length, that stimuli presented in close temporal proximity tend to be assimilated or averaged. Thus, one line length presented in immediate conjunction with another will be judged as being more similar to the preceding stimulus than will the same line presented in isolation. On the other hand, a stimulus that is temporally separated from an initial stimulus (i.e., presented after a delay) tends to show contrast; that is, it is judged as being more different from the previous stimulus than the same line presented alone.

Now consider the fact that any visual array that subtends a suprafoveal visual angle leads to sequential information input of the parts of the array, since the observer apprehends the array in a series of successive fixations over the figure. The pool-and-store model simply proposes that the observer uses each single fixation or glance to garner a global impression of one part of the stimulus array. Thus, if more than one visual extent is present in a single glance, the model proposes that the extents will be pooled or averaged. On the other hand, when multiple glances are required for more than one extent to be seen, the information from early glances must be held in storage to be used later in the synthesis of the entire stimulus array into the final 
percept. Under these circumstances, it becomes important for the observer to distinguish between each new piece of stimulus information and the information from previous glances already held in storage. The pool-andstore model proposes that, in order to distinguish between new and old information, the observer extracts and emphasizes the differences between the information contained in each new glance and the information already held in storage from previous glances. Thus, if one visual extent is seen in the first glance and a different visual extent is seen in a second glance, the differences between them will be emphasized and contrast will result. [Mornaga (1935, in Oyama, 1960) may have been thinking along similar lines when he concluded that assimilation in the Delboeuf illusion occurs when the two circles are seen as a whole and contrast occurs when they are received as separate percepts.]

It is quite easy to translate these proposed mechanisms into predictions based upon spatial relations within a given stimulus. When the parts of the stimulus are spatially proximal, they are presumably sampled in a single glance, and hence are pooled or assimilated. On the other hand, when the parts of the stimulus are far away from each other spatially, they must be sampled in successive glances, with contrast between them the direct consequence. Let us now apply this analysis to the illusion configurations used in the set of studies described above and see if the predictions are consistent with the obtained results.

Because in the overestimated, or assimilation, portion of the Delboeuf illusion, the two circles are always close together, it seems likely that the inducing element is in view whenever the observer is looking at the test element. According to the model, the sizes of the two circles should be pooled or assimilated. This implies that the size of the inner circle will be overestimated and the size of the outer circle will be slightly underestimated, which is the result usually obtained (see Coren \& Girgus, 1978, for a review). In the underestimated, or contrast, portion of the Delboeuf, a different situation pertains. In this configuration, the two circles are sufficiently far apart that the inducing circle is probably never in foveal view when the observer is looking at the test circle. Thus, the test and inducing circles are seen only in successive glances, which should lead to an exaggeration in the difference in size between them and hence to the perceived contrast distortion.

The notion that the Ebbinghaus illusion can be thought of as an assimilation/contrast illusion is supported by the data described in this report. This fits well with the work of previous investigators (Cooper \& Weintraub, 1970; Girgus, Coren, \& Agdern, 1972) who have suggested that the Ebbinghaus illusion is actually a variant of the Delboeuf illusion, with the critical distance between inner and outer circles formed either by the distance between the central circle and the centers of the surrounding circles or by the distance between the central circle and the outer edges of the surrounding circles. When one thinks about it this way, it is clear that the central circle and the centers or outer edges of the surrounding circles are much more likely to be seen in a single glance in the assimilation, or overestimated, portion of the Ebbinghaus illusion when the context circles are small and much more likely to be seen only in successive glances in the contrast, or underestimated, half when they are large.

In the Ponzo illusion, the critical dimensions pertain to the relationship between elements lying near the horizontal extent to be judged. It is unlikely that the test line near the apex of the angle could be viewed without some part of the converging sides also being in view. According to the model, this should lead to a pooling of the length of the test line with the distance between the sides of the angle and hence to overestimation of the test line. When the test line is placed far from the apex in the wider part of the angle, it is unlikely that this test element and the sides of the angle will be seen together in a single glance. Under these circumstances, the length of the test line should be contrasted with the horizontal distance between the sides of the angle, and thus underestimated.

It seems reasonable to expect that any stimulus information received in a single glance, and thus pooled or assimilated, is not subject to cognitive recalibration over repeated glances, provided that the test and inducing extents critical for the assimilation process always occur together in every glance. On the other hand, stored or contrasted extents presumably are subject to such recalibration during free inspection, since there is already some cognitive manipulation of the differences between them during the storage and comparison processes. This is supported by the Hoenig (1972) data on the MuellerLyer illusion, in which data no decrement was found under free inspection when the size of the illusion figure was subfoveal.

There are other data already extant in the literature that seem to provide indirect support for the pool-andstore model of assimilation/contrast processing. For both the apparently longer and apparently shorter half of the Mueller-Lyer illusion, the model would predict an assimilation or pooling of the length of the shaft with the average locus of the points that make up the wings, since the ends of the shaft can never be seen without a simultaneous view of the wings. However, as the ends of the shaft are separated from the wings, both halves of the illusion should switch from assimilation to contrast effects. This phenomenon has, in fact, been reported by Pollack and Chaplin (1964) and by Fellows (1968).

It is important to recognize that intercontour distance may not always be the relevant factor in determining whether assimilation or contrast will occur. For instance, in the Ebbinghaus configuration used in Experiment 2, the distance between the test and inducing circles remained fixed at $43 \mathrm{~min}$ of visual angle. 
However, the relevant factor involved in the assimilation or contrast illusion is, of course, the circle sizes (and their effect on successive versus simultaneous comparison of elements), not simply the absolute contour proximity. Thus, for assimilation to occur in the Ebbinghaus illusion, each glance should include a large proportion of the circles being compared. In the assimilation configuration used in this study, the distance between the center of the test circle and the center of each inducing circle was $2 \mathrm{deg}$ and hence easily foveal. The distance between the center of the test circle and the center of each inducing circle in the contrast configuration, on the other hand, was $4.5 \mathrm{deg}$, which is larger than the foveal extent. It is the critical elements, rather than merely the closest aspect of any contour, that must occur in the same glance.

Such an analysis suggests that any means of separating the test and inducing elements, or of bringing them together, whether it involves spatial separation or temporal separation, should produce predictable results according to the model. In this context, further evidence in support of the pool-and-store model comes from experiments that have used temporal separation of test and inducing elements that are normally seen together in a single glance. For example, in the Delboeuf illusion, one might present first the surrounding circle alone and then the test circle alone. Under these circumstances, the model would predict that only contrast effects could be found. This prediction has been confirmed by Cooper and Weintraub (1970) for the Delboeuf illusion, by Pollack (1964) for the Mueller-Lyer illusion, and by Brigell and Uhlarik (1979) for a variant of the MuellerLyer illusion.

The pool-and-store model has several advantages over more descriptive models that have been previously proposed to explain assimilation/contrast illusions (Fisher, 1969, 1973; Helmholtz, 1866/1962; Helson, 1964; Pressey, 1971; Restle, 1971a). To begin with, it proposes that assimilation and contrast illusions result from the same general processes by which pattern information is extracted from the visual field and entered into storage for later use. Second, the same model that predicts the illusory effect may be used to predict the relative plasticity of the distortion under prolonged inspection. Such a model clearly has implications for several problems in general pattern perception, as well as for illusory effects. At the very least, the model implies that the critical dimension in assimilation/ contrast illusion configurations is not the relative size of test and inducing elements, but rather the absolute size, in terms of visual angle.

\section{REFLRENCES}

Benussi, V. Zur Psychologie des Gestalterfassens (die Millerlyersche Figur). In A. Meinong (Ed.), Untersuchurgen zur Gegenstands Theorie und Psychologle. Leipzig: Burth, 1904.
Brigeld, M., \& UhlariK, J. The relational determination of length illusions and length aftereffects. Perception, 1979, 8, 187.197.

Brunot, C. Les illusions optiques. Revue Scientifique Paris, 1893, 3 Ser., 52, 210-212.

Cooper, L. A., \& Weintraub, D. J. Delboeuf-type circle illusions: Interactions among luminance, temporal characteristics and inducing-figure variations. Journal of Experimental Psychology, 1970, 85, 15-82.

Conen, S. A size contrast illusion without physical size difference. American Journal of Psychology, 1971, 84, 565-566.

Coren, S., \& Giraus, J. S. Density of human lens pigmentation: In vivo measures over an extended age range. Vision Research, 1972, 12, 343-346. (a)

Coren, S., \& Girgus, J. S. Illusions decrement in intersecting line figures. Psychonomic Science, 1972, 26, 108-110. (b)

Conen, S., \& Giraus, J. S. Transfer of illusion decrement as a function of perceived similarity. Journal of Experimental Psychology, 1974, 102, 881-887.

Conen, S., \& Ginous, J. S. Seeing is deceiving: The psychology of visual illusions. Hillsdale, N.J: Erlbaum, 1978.

Coren, 3., Gingus, J. S., Emlichuan, H., \& Hakstian, A. R. An empirical taxonomy of visual illusions. Perception \& Psychophysics, 1976, 20, 129-137.

Conen, S., \& Hoenia, P. Eye movements and decrement in the Oppel-Kundt illusion. Perception \& Psychophysics, 1972, 12, 224-225.

Conen, S., \& Miluer, J. Size contrast as a function of figural similarity. Perception \& Psychophysics, 1974, 16, 355-357.

Dewar, R. E. Distribution of practice and the Muiller-Lyer illusion. Perception \& Psychophysics, 1968, 3, 246-248.

Enlebacher, A., \& Sexular, R. Explanation of the MuellerLyer illusion: Confusion theory examined. Journal of Experimental Psychology, 1969, 80, 462-467.

Fellows, B. J. The reverse Mueller-Lyer illusion and "enclosure." British Journal of Psychology, 1968, 59, 369-372.

Fisher, G. H. Towards a new explanation for the geometrical illusions. 1. The properties of contours which induce illusory distortion. British Journal of Psychology, 1969, 60, 170-187.

Frsher, G. H. Towards a new explanation for the geometrical illusions. II. Apparent depth or contour proximity? British Journal of Psychology, 1973, 44, 607-621.

Girous, J. S., Coren, S., \& Agdern, M. The interrelationship between the Ebbinghaus and Delboeuf illusions. Journal of Experimental Psychology, 1972, 95, 453-455.

Girous, J. S., Coren, S., Durant, M., \& Porac, C. The assessment of components involved in illusion formation using a long term decrement procedure. Perception \& Psychophysics, 1975, 18, 144-148.

Helmholtz, H. von. Treatise on physiological optics (J. P. C. Southall, trans.). New York: Dover, 1962 . (Originally published, 1866.)

Hzison, H. Adaptation level theory: An experiment and systematic approach to behavior. New York: Harper, 1964. (a)

Hezson, H. Current trends and issues in adaptation-level theory. American Psychologist, 1964, 19, 26-38. (b)

Hoznia, P. The effects of eye movements, fixation and figure size on decrement in the Mueller-Lyer illusion. Unpublished doctoral thesis, The New School for Social Research, 1972.

JuDD, C. H. Practice and its effects on the perception of illusions. Psychological Review, 1902, 9, 27-39.

Massano, D. W., \& ANDerson, N. H. Judgmental model of the Ebbinghaus illusion. Journal of Experimental Psychology, $1971,81,147-151$.

Mountsoy, P. T. Effects of exposure time and intertrial interval upon decrement to the Mueller-Lyer illusion. Journal of Experimental Psychology, 1958, 56, 97-102.

OYAMA, T. Japanese studies on the so-called geometrical-optical illusions, Psychologia, 1960, 3, 7-20. 
Polica, R. H. Simultaneous and successive presentation of elements of the Mueller-Lyer figure and chronological age. Perceptual and Motor Skills, 1964, 19, 303-310.

Pollack, R. H., Chaplin, M. R. Effects of prolonged stimulation by components of the Mueller-Lyer figure upon the magnitude of the illusion. Penceptual and Motor Skills, 1964, 18, 377-382.

Porac, C., Coren, S., Giraus, J. S., \& Verde, M. Visualgeometric illusions: Uni-sexed phenomena. Perception, 1979, $8,401-412$.

Presser, A. W. The assimilation theory applied to a modification of the Mueller-Lyer illusion. Perception \& Psycho physics, 1970, 8, 411-412.

Presszy, A. W. An extension of assimilation theory to illusions of size, area and direction. Perception \& Psychophysics, 1971, 9, 172-176.

Quina, K., \& Pollack, R. H. Effects of test line position and age on the magnitude of the Ponzo illusion. Penception \& Psychophysics, 1972, 12, 253-256.

Restr.e, F. Visual illusions. In M. H. Appley (Ed.), Adaptationlevel theory. New York: Academic Press, 1971. (a)
Restze, F. Instructions and the magnitude of an illusion: Cognitive factors in the frame of reference. Perception \& Psychophysics, 1971,9,31-32. (b)

Restue, F., \& Merryman, C. T. Distance and an illusion of length of line. Journal of Experimental Pyychology, 1968, 81, 297-303. (b)

Swith, R. A compleat system of opticks. (2 vols.). Cambridge: Crownfield, 1738.

WARD, L. M., \& Lockhend, G. R. Sequential effects and memory in category judgments. Journal of Experimental Psychology, 1970, 84, 27-34.

WARD, L. M., \& Lockhend, G. R. Response system processes in absolute judgment. Perception \& Psychophysics, 1971, 9, 73-78.

WUNDr, W. [Lectures on human and animal psychology] (J. E. Creighton \& E. B. Titchener, trans.). London: Swan Sonnenschein, 1894.

(Manuscript received August 17, 1981; revision accepted for publication September 21, 1982.) 